Journal of Animal and Veterinary Advances $10(6): 791-795,2011$

ISSN: $1680-5593$

(C) Medwell Journals, 2011

\title{
Culture of Chicken Embryonic Stem Cells in Buffalo Rat Liver 3A Cells (BRL-3A) Conditioned Medium
}

\author{
${ }^{1}$ Li-Ying Geng, ${ }^{2}$ Chuan-Sheng Zhang, ${ }^{2}$ Shen Ping, ${ }^{3} \mathrm{Li}$-Xin DU, ${ }^{2}$ Zheng-Zhu Liu, \\ ${ }^{2} \mathrm{Zhi}-\mathrm{Xin} \mathrm{Fu},{ }^{2} \mathrm{Chen}$ Juan, ${ }^{2} \mathrm{Q}$ ing-Hui Jia and ${ }^{2} \mathrm{Xie}-$ Rong Liu \\ ${ }^{1}$ Department of Life Science, ${ }^{2}$ Department of Animal Science, \\ Hebei Normal University of Science and Technology, 066600 Changli, China \\ ${ }^{3}$ National Center for Molecular Genetics and Breeding of Animal, \\ Institute of Animal Science, Chinese Academy of Agricultural Sciences, \\ 100193 Beijing, China
}

\begin{abstract}
BRL-3A conditioned medium combined with basic Fibroblast Growth Factor (bFGF), Insulin-like Growth Factor (IGF), Stem Cell Factor (SCF) and STO cells are used for the culture of the feeder layer and ES (Embryonic Stem) cell clones. This study is done by isolation of chicken X-stage blastodermal cells. The putative ES cell clone features a typical bird's nest structure, PAS and AKP-positive and strongly positive for SSEA-1. It is able to form embryoid bodies and multiple types of cells as well as participates in embryonic development and feather color chimera formation through in vitro differentiation.
\end{abstract}

Key words: Chicken, BRL-3A cells, in vitro culture, ES cell, embryonic stem, cell clones

\section{INTRODUCTION}

The embryonic germ cells originating from the primordial germ cells and the cells from the inner cell mass are collectively called Embryonic Stem (ES) cells which have the ability to proliferate and differentiate into all types of cells and tissues. These cells have broad application prospects in basic research as well as in the research and development of transgenic animals (Keefer et al., 2007). Since, the establishment of the first ESC lines in mice there have been numerous reports of ESC lines establishment in other species (Evans and Kaufman, 1981; Brons et al., 2007). Still, no ESCs from other animals as from the mice have been demonstrated to colonize the germ line. Further more, the derivation and maintenance of ESCs from other species other than the mouse is still difficult. This may primarily be due to the lack of a suitable culture conditions that can effectively inhibit differentiation of isolated ESCs from these species including chicken.

The ES cells of chicken are mainly isolated from Xstage blastodermal cells due to their typical reproductive features (Park et al., 2003; Van de Lavoir et al., 2006). Pain et al. (1996) have reported the isolation and culture system for chicken ES cells (Pain et al., 1996). In early studies, Buffalo Rat Liver Cells-Conditioned Medium (BRL-CM) was used to establish a culture system for chicken ES cells but only ES like cells were obtained after short-term subculture (Meng et al., 2008).

This study further optimizes the conditions for the culture of chicken blastodermal cells and makes detailed identifications of the ES cell features of the ES cell clones obtained. It sets a solid foundation for the infinite proliferation of the chicken ES cell line.

\section{MATERIALS AND METHODS}

Experiment materials: Hatching eggs were obtained from a representative poultry farm in Hebei Normal University of Science and Technology. They were hatched at $38^{\circ} \mathrm{C}$ and $60 \%$ relative humidity. The BRL-3A (rat liver cell line) cells and STO (mouse embryonic fibroblast cell line) cells were purchased from the Institute of Biochemistry and Cell Biology, Shanghai Institutes for Biological Science, Chinese Academy of Sciences.

Media and reagents: High-glucose Dulbecco's Modified Eagle's Medium (DMEM), Fetal Bovine Serum (FBS), sodium pyruvate, Phosphate-Buffered Saline (PBS), trypsase, chicken serum, $\beta$-mercaptoethanol (purchased

Corresponding Author: Chuan-Sheng Zhang, Laboratory of Molecular Genetic and Animal Breeding, Department of Animal Science, Hebei Normal University of Science and Technology, 066600 Changli, China 
from Gibco); mouse Leukemia Inhibitor Gene (LIF), bFGF, SCF, IGF-I, mitomycin C (purchased from Sigma); SSEA-1 and SSEA-4 antibodies; Horseradish Peroxidase (HRP)-conjugated goat anti-mouse IgG.

Preparation of feeder layer: Mature STO cells were adopted and the original culture solution was discarded. After rinsing with PBS, DMEM with $10 \mu \mathrm{g} \mathrm{mL}-1$ mitomycin $\mathrm{C}$ was added and the resulting solution was incubated at $37^{\circ} \mathrm{C}$ for $2 \mathrm{~h}$. Then, the culture solution containing the mitomycin $\mathrm{C}$ was discarded and the cells were rinsed with PBS. The cells were then digested with trypsase into a single cell suspension. The cell concentration was subsequently adjusted to $5 \times 10^{5} \mathrm{~mL}^{-1}$. The cell suspension was placed into a culture dish without gelatin and cultured at $37^{\circ} \mathrm{C}$ under a $5 \% \mathrm{CO}_{2}$ atmosphere with saturated humidity.

Preparation of BRL-CM: The BRL-3A cells were cultured in DMEM culture solution with $10 \%$ FBS. The culture solution was collected 3 days later and stored at $-20^{\circ} \mathrm{C}$. Solution samples were collected 3 times and then filtered with $0.22 \mu \mathrm{m}$ membrane after mixing. Then the $\mathrm{pH}$ was adjusted to 7.5. Composition of different culture systems: Solution I: DMEM+10\% FBS; Solution II: BRL-CM; Solution III: BRL-CM+4:1 mixture of 10\% FBS and DMEM with one or a combination of the following growth factors: $1 \times 10^{6} \mathrm{U} \mathrm{L}^{-1} \mathrm{LIF}, 1 \times 10^{4} \mathrm{ng} \mathrm{L^{-1 }}$ Stem Cell Factor $(\mathrm{SCF})$, $1 \times 10^{4}$ ng $\mathrm{L}^{-1}$ Fibroblast Growth Factor (FGF) and $1 \times 10^{4} \mathrm{ng} \mathrm{L}^{-1}$ Insulin-like Growth Factor 1 (IGF-1).

The following substances are added, respectively to the foregoing solutions to the corresponding final concentrations: $1 \%$ non-essential amino acid, $2 \mathrm{mmol} \mathrm{L}{ }^{-1}$ glutamine, $1 \mathrm{mmol} \mathrm{L}^{-1}$ sodium pyruvate, $0.1 \mathrm{mmol} \mathrm{L}^{-1} \beta$-mercaptoethanol, $1 \mathrm{mmol} \mathrm{L}{ }^{-1} \mathrm{HEPES}$, $100 \mathrm{IU} \mathrm{mL}^{-1}$ penicillin, $100 \mathrm{mg} \mathrm{mL}^{-1}$ streptomycin and $2 \%$ chicken serum. System I: feeder-free+Solution I; System II: feeder-free+Solution II; System III: feeder-free+Solution III; System IV: STO feeder layer+Solution II; System V: STO feeder layer+Solution III.

Isolation and culture of $\mathbf{X}$-stage blastodermal cells: The fresh hatching eggs of Shouguang chickens were selected. The blastodermal zonae pellucidae were isolated aseptically via the filter study loop method after sterilization, rinse with PBS and then centrifugated at $100 \times \mathrm{g}$ for $5 \mathrm{~min}$. The cell mass was collected and prepared into a single cell suspension with $0.25 \%$ trypsin and $0.02 \%$ EDTA after trypsinization. The digestive solution was then replaced with ES cell culture solution which was inoculated after resuspension into the culture plate covered with feeder layer. The incubation density was one blastodermal per $\mathrm{mL}$ culture solution. Half of the medium was changed every $24 \mathrm{~h}$. On the 4 th- 5 th day, after the ES-like cell colony was formed, the cells around the colony tended to differentiate i.e., subculture. The incubation density of ES cells was $5 \times 10^{4} \mathrm{~mL}^{-1}$. Half of the medium was changed every $12 \mathrm{~h}$. The subculture was conducted in the same way.

\section{Identification of chicken ES cells Identification by Periodic Acid-Schiff(PAS) staining and Alkaline Phosphatase (AKP) staining: The 6th} generation mature chicken ES cell colonies were selected and subjected to PAS as well as alkaline phosphatase chromogen staining using staining kits according to the manufacturer's instructions. The cells were then observed and photographed.

Immunocytochemical stain: SSEA-1 and SSEA-4 immunofluorescence staining were conducted on the 3rd generation mature chicken ES cells according to the kit manufacturer's instructions. The cells were then observed under a fluorescence microscope and photographed.

In vitro and in vivo differentiation experiments: The 6th generation ES cells were selected and digested under a slightly shortened digestion time. The cells were blown gently to divide them into many small cell masses and transferred into a culture dish $(35 \mathrm{~mm})$. No inhibitory factors were added into the culture solution. The culture was blown daily with a blowpipe and the formation of embryoid bodies was observed. The 6th generation mature ES cell clones were selected and prepared into a single-cell suspension after digestion. The cell density was then adjusted to $10^{6}-10^{7} \mathrm{~mL}^{-1}$ and then $2-5 \mu \mathrm{L}$ of the cell suspension was injected into the subgerminal cavity of the receptor and transferred into an eggshell. The opening was then sealed and the egg was incubated until the chick hatched.

Test of the genes related to chicken ES cell pluripotency: The total RNA of the cells was extracted via the Trizol method and was cDNA synthesized with avian myeloblastosis virus reverse transcriptase and Oligo (dT) 18 primer. The primer was designed and amplified using Primer 5.0. The primer sequence and fragment length of the pluripotent genes are shown in Table 1.

The genes were amplified via PCR based on the template of the synthetic cDNA according to the following parameters: initial denaturation at $95^{\circ} \mathrm{C}$ for $5 \mathrm{~min}$; denaturation at $95^{\circ} \mathrm{C}$ for $40 \mathrm{sec}$; annealing at $58^{\circ} \mathrm{C}$ for $30 \mathrm{sec} ; 35 \mathrm{cycles}$ of extension at $72^{\circ} \mathrm{C}$ for $45 \mathrm{sec}$; final extension at $72^{\circ} \mathrm{C}$ for $10 \mathrm{~min}$. Then, $5 \mu \mathrm{L}$ amplified product was electrophoresed on $2 \%$ agarose gel observed with a gel imaging system and photographed. 


\begin{tabular}{llcc}
\multicolumn{5}{c}{ Table 1: Primer sequences for RT-PCR analy sis of target genes } \\
\hline \multicolumn{1}{c}{$\begin{array}{c}\text { Tmp } \\
\text { Primer }\end{array}$} & \multicolumn{1}{c}{ PCR } \\
Primer sequence (5'-3') & $\left({ }^{\circ} \mathrm{C}\right)$ & product (bp) \\
\hline cPouV & 5'-GTTGTCCGGGTCTGGTTCT-3' & & \\
& 5'-GTGGAAAGGTGGCATGTAGAC-3' & 58 & 189 \\
cNanog & 5'-CAGCAGACCTCTCCTTGACC-3' & & \\
& 5'-TTCCTTGTCCCTCTCTCACC-3' & 58 & 187 \\
ERNI & 5'-GATCTAGATCCTCAAATGAAT-3' & & \\
& 5'-TCTTGGGCAACCTCTCCCC-3' & 55 & 594 \\
\hline
\end{tabular}

Effects of different growth factors and their combination on the culture of ES cells: The BRL-CM was treated with one or a combination of bFGF, IGF, SCF and IGF-1 in different culture media. Chicken blastodermal cells were cultured on an STO cell feeder layer. The generation of positive cell clones was visualized by AKP staining ( 3 wells for each group, repeated 3 times). The effects of the different growth factors and its combination on the culture of ES cells were observed.

Data analysis: The experimental data is expressed in percent and the significance of difference was analyzed through t-test.

\section{RESULTS AND DISCUSSION}

Observation and identification of growth of BRL-3A cells and chicken ES cells: The BRL-3A cells grew in adherence as irregular polygons. The cells grew fast and a paving stone-like pattern was formed after covering with the cells (Fig. 1a). In the feeder-free System I, the blastodermal cell adhered quickly. The flat single-layer polygonal cells and small multiple-layer island cells were formed after culture for $48 \mathrm{~h}$ (Fig. 1b). In feeder-free Systems II and III, the cell adherence slows down and cell islands were formed (Fig. 1c). In Systems IV and V with feeder layers, the blastodermal cells formed ES cell clones. The ES cell clones obtained in System IV remained undifferentiated without subculture. In contrast, the ES cell clones obtained in System V only reached the 8th generation. Chicken ES cell clones grew adherence to the culture plate and exhibited apophysis even edges and was shaped like a bird's nest and mound (Fig. 1d and e). During the subculture, ES cells formed many smaller masses. At $48 \mathrm{~h}$ they coalesced and grew into the typical bird's nest formation (Fig. 1f). The chicken ES cell clones were purple-crimson after PAS staining (Fig. 1g) and brown-yellow after AKP staining (Fig. $1 \mathrm{~h}$ ). The cells of the feeder layer were not stained. The chicken ES cell colony of the 6th generation was SSEA-1 positive (Fig. 1i) and SSEA-4 negative (Fig. 1j). In the differentiation culture system, the embryoid bodies (Fig. 1k) were formed after suspension culture for 2-4 days. A feather-colored chimera was obtained after it was injected into the subgerminal cavity of the hatching egg (Fig. 1).
Test of genes related to cell multipotency: The RT-PCR results of $\mathrm{cPouV}$, cNanog and ERNI mRNA expression indicate that $\mathrm{cPouV}, \mathrm{cNanog}$ and ERNI are genes related to the significant expression of chicken ES-like cell clone multipotency. The results are shown in Fig. 2.

Effect of different growth factors and their combination upon culture results of ES cells: During the experiment, LIF, SCF, bFGF and other growth factors were added into the BRL-CM culture solution at different ratios. Afterward, ES-like cell proliferation was observed ( 3 wells per group, for 3 replicates). The results are shown in Fig. 3. The results indicate that SCF and bFGF may promote the growth of ES cells at different degrees; the chicken ES-like cell AKP positive clone resulting from the combination of four growth factors differed significantly from those by any 1 growth factor or a combination of any 3 growth factors $(\mathrm{p}<0.01)$. The scattered $\mathrm{X}$-stage blastodermal cells were cultured in different systems. The blastodermal cells differentiated into flat single-layer cells and multiple-layer island cells in the feeder-free condition and no ES cell clone was obtained. The ES cell colony was obtained when a feeder layer was used. Such a result is consistent with that of previous studies i.e., the chicken ES cells depend on the feeder layer (Pain et al., 1996). Notably, the ES-like cells obtained from System IV differentiates fast without subculture. This indicates that the BRL-CM and STO feeder layer interact with each other, providing the microenvironment or the growth factors necessary for the formation of the chicken ES clones. However, they are not sufficient to promote cell proliferation or maintaining the undifferentiated state.

Presently, scholars mainly solve problems concerning proliferation and differentiation by adding LIF and other growth factors and the undifferentiated state of chicken ES cells could be maintained by activating LIF-gp130-STAT3 (Horiuchi et al., 2004, 2006; Yamashita et al., 2006). They also found that the combination of IGF-I, SCF and other growth factors may promote the isolated culture and subculture of chicken ES cells. Combination of BRL-CM with the four growth factors LIF, SCF, IGF and bFGF (i.e., System V) may increase the positive cloning efficiency of chicken ES cells $(\mathrm{p}<0.01)$ and prolong in vitro culture time. When only one growth factor was used, the chicken ES cells differentiated significantly even though the BRL-CM and the STO cell feeder layer were used. When BRL-CM was combined with LIF only, a clone was formed but the process was too slow and the ES cell clone only reached the 3rd generation (Meng et al., 2008). The results indicate that the 4 growth factors may be necessary for the growth of chicken ES cells. Efficient interaction and complementation among the growth factors and between the growth factors and BRL-CM may achieve desirable results. 


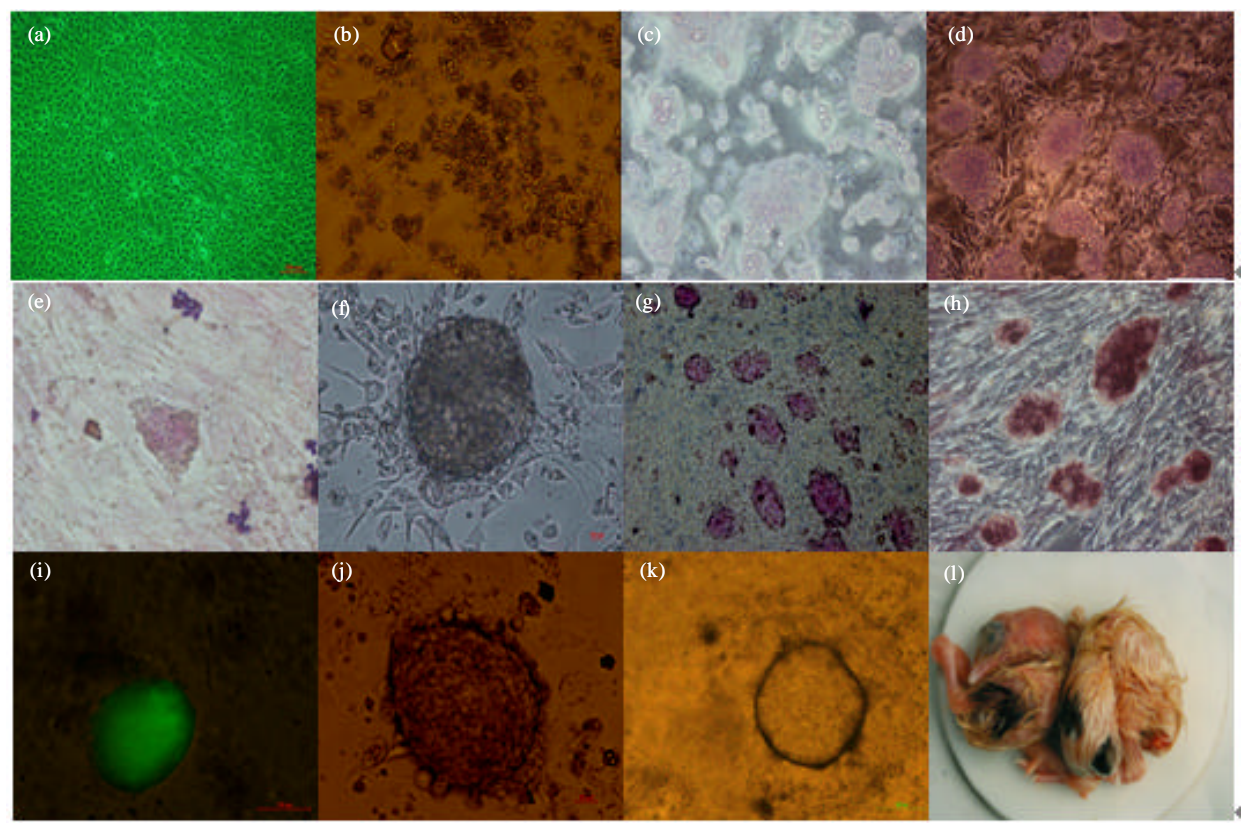

Fig. 1: Derivation and characteristics of ES cells obtained from chicken X-stage blastodermal cells; a) BRL-3A cells $\times 200$; b) blastodermal cell cultured in feeder-free System I $\times 400$; c) t blastodermal cell cultured in feeder-free System II and III $\times 400$; $\mathrm{d}$ and e) Chicken ES cell clones colony $\times 400 ;$ f) the 5 passages ES cells $\times 250$; g) PAS staining ES Cells $\times 250$; h) AKP staining ES cells $\times 250$; i) SSEA-1 light positive $(400 \times)$; j) SSEA-4 light negative $(400 \times)$; k) Embryoid bodies formed in suspension after 6 days of culture $(100 \times) ; 1)$ the feather-colored chimera

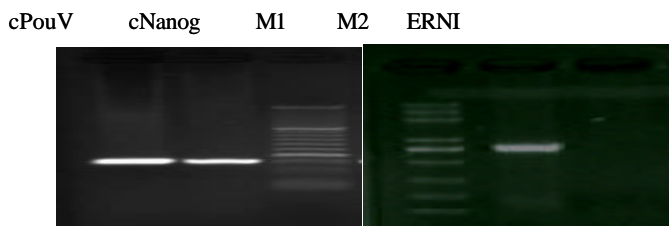

Fig. 2: Expression of cPouV, cNanog and ERNI in chicken ES cells, M1/50 bp ladder, M2/100 bp ladder

The chicken ES cells obtained from System V were identical to those reported in previous investigations in many respects such as PAS and AKP positive, SSEA-1 positive, PouV, Nanog and ERNI tests that express ES cell multipotency or the ability to differentiate into embryoid body-like structures, participation in early embryonic development and formation of feather color chimera (Lavial et al., 2007; Lavial and Pain, 2010; Acloque et al., 2001).

However, this study was only able to obtain 8th generation ES cells. Yang et al. has cultured chicken ES cell in vitro into the 20th generation under similar, conditions.

The reason for such a difference probably lies in 2 aspects. First, the ability of BRL-3A cells to secrete factors decreases due to repeated freezing and long storage and the conditioned medium was affected accordingly; second, the concentration of different growth factors requires further optimization. Overall, this

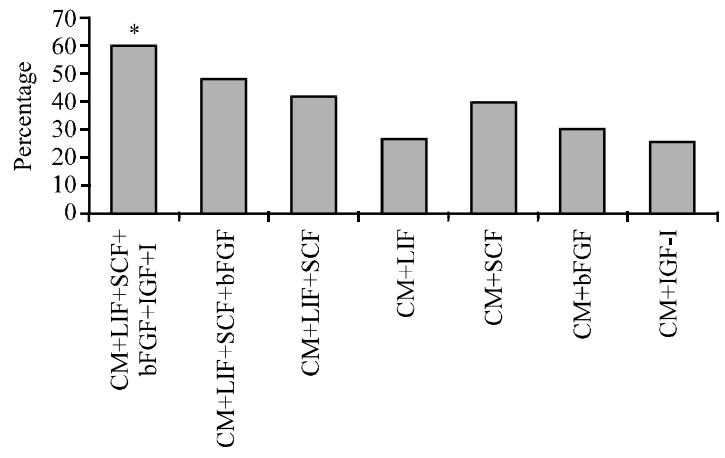

Fig. 3: Percentage of AKP positive colonies with the treatment of different cytokines

study has studied the conditions for the culture of chicken ES cells with BRL-CM and set a solid foundation for the infinite culture of chicken ES cell lines.

\section{CONCLUSION}

The results of the RT-PCR and gene sequencing indicate that the expression of PouV, Nanog and ERNI (i.e., the genes related to pluripotency of stem cells) are significant in chicken ES cells. The BRL-3A conditioned medium combined with growth factors bFGF, IGF, SCF and LIF may promote the formation of chicken ES cell clones. 


\section{ACKNOWLEDGEMENTS}

This research was supported by key projects in the National Science and Technology pillar program during the 11th 5 year plan period (No. 2008 BADB2B04-8-4), Natural Science Foundation of Hebei Province (No. C2008001308) and National Science Foundation for Youths (No. 31001003).

\section{REFERENCES}

Acloque, H., V. Risson, A.M. Birot, R. Kunita, B. Pain and J. Samarut, 2001. Identification of a new gene family specifically expressed in chicken embryonic stem cells and early embryo. Mechanisms Dev., 103: 79-91.

Brons, I.G.M., L.E. Smithers, M.W.B. Trotter, P. RuggGunn and B.W. Sun et al., 2007. Derivation of pluripotent epiblast stem cells from mammalian embryos. Nature, 448: 191-195.

Evans, M.J. and M.H. Kaufman, 1981. Establishment in culture of pluripotential cells from mouse embryos. Nature, 292: 154-156.

Horiuchi, H., A. Tategaki, Y. Yamashita, H. Hisamatsu and M. Ogawa et al., 2004. Chicken leukemia inhibitory factor maintains chicken embryonic stem cells in the undifferentiated state. J. Biol. Chem., 279: 24514-24520.

Horiuchi, H., S. Furusawa and H. Matsuda, 2006. Maintenance of chicken embryonic stem cells in vitro. Methods Mol. Biol., 329: 17-34.

Keefer, C.L., D. Pant, L. Blomberg and N.C. Talbot, 2007. Challenges and prospects for the establishment of embryonic stem cell lines of domesticated ungulates. Anim. Reprod. Sci., 98: 147-168.
Lavial, F. and B. Pain, 2010. Chicken embryonic stem cells as a non-mammalian embryonic stem cell model. Dev. Growth Differentiation, 52: 101-114.

Lavial, F., H. Acloque, F. Bertocchini, D.J. Macleod and S. Boast et al., 2007. The Oct4 homologue PouV and Nanog regulate pluripotency in chicken embryonic stem cells. Development, 134: 3549-3563.

Meng, C.H., C.S. Zhang, N.N. Yang, Z.Y. Wang, S.X. Cao and L.X. Du, 2008. Preliminary study on culture of chicken embryonic stem cells by buffalo rat liver cells conditioned medium. Chinese J. Cell Biol., 30: $532-536$.

Pain B., M.E. Clark, M. Shen, H. Nakazawa, M. Sakurai, J. Samarut and R. J. Etches, 1996. Long-term in vitro culture and characterization of avian embryonic stem cells with multiple morphogenetic potentialities. Development, 122: 2339-2348.

Park, T.S., Y.H. Hong, S.C. Kwon, J.M. Lim and J.Y. Han, 2003. Birth of germline chimeras by transfer of chicken Embryonic Germ (EG) cells into recipient embryos. Mol. Reprod. Dev., 65: 389-395.

Van de Lavoir, M.C., C. Mather-Love, P. Leighton, J.H. Diamond and B.S. Heyer et al., 2006. High-grade transgenic somatic chimeras from chicken embryonic stem cells. Mechanisms Dev., 123: 31-41.

Yamashita, Y., A. Tategaki, M. Ogawa, H. Horiuchi and K. Nishida et al., 2006. Effect of novel monoclonal antibodies on LIF-induced signaling in chicken blastodermal cells. Dev. Comparative Immunol., 30: $513-522$. 